\title{
Application value of double-layer spectral detector CT in differentiating central lung cancer from atelectasis
}

\author{
Li-Juan Wen ${ }^{1 \#}$, Qi-Yu Zhao ${ }^{2 \#}$, Yue-Hui Yin ${ }^{3}$, Bing Wang ${ }^{1}$, Ming-Xin Fan ${ }^{1}$, Yuan-Gang Qi ${ }^{2}$, Qing Zhang ${ }^{1}$ \\ ${ }^{1}$ Department of Radiology, The Third Affiliated Hospital of Shandong First Medical University (Affiliated Hospital of Shandong Academy of \\ Medical Sciences), Jinan, China; ${ }^{2}$ Department of Radiology, Shandong Cancer Hospital and Institute, Shandong First Medical University and \\ Shandong Academy of Medical Sciences, Jinan, China; ${ }^{3}$ Department of Radiology, Weifang People's Hospital, Weifang, China \\ Contributions: (I) Conception and design: Q Zhang, YG Qi; (II) Administrative support: Q Zhang, YG Qi; (III) Provision of study materials or \\ patients: YG Qi; (IV) Collection and assembly of data: LJ Wen, QY Zhao, YH Yin, B Wang, MX Fan; (V) Data analysis and interpretation: LJ Wen, \\ QY Zhao, YH Yin; (VI) Manuscript writing: All authors; (VII) Final approval of manuscript: All authors. \\ \#These authors contributed equally to this work. \\ Correspondence to: Qing Zhang. Department of Radiology, The Third Affiliated Hospital of Shandong First Medical University (Affiliated Hospital \\ of Shandong Academy of Medical Sciences), 38\# Wuyingshan Road, Jinan 250031, China. Email: radiozq@qq.com; Yuan-Gang Qi. Department of \\ Radiology, Shandong Cancer Hospital and Institute, Shandong First Medical University and Shandong Academy of Medical Sciences, 440\# Jiyan \\ Road, Jinan 250117, China. Email: qiyuangang2004@126.com.
}

Backgroundk Central lung cancer with obstructive atelectasis is very common in clinical practice. Determination of the tumor borderline is important. Conventional computed tomography (CT) alone may not be sufficiently accurate to distinguish central lung cancer from obstructive atelectasis. Spectral CT can improve the soft-tissue resolution greatly. In this study, we evaluated the application value of double-layer spectral detector CT in differentiating central lung cancer from atelectasis.

Methods: A total of 51 patients $(37$ males) with pathologically confirmed central lung cancer accompanied by atelectasis were enrolled. The rates of differentiation between tumors and atelectasis were retrospectively analyzed using conventional CT and three types of spectral images (40 keV virtual monoenergetic imaging, iodine density map, and their fusion image) of unenhanced scans as well as arterial and venous phases. Cochran's Q test and Friedman test were used to compare the differentiation rates and the maximal diameters of the tumors in each image.

Results: Among the 51 cases, conventional CT, $40 \mathrm{keV}$ monoenergetic, iodine density, and their fusion images of the venous phase were successful in differentiating tumors from atelectasis in 17 (33.33\%), 35 (68.63\%), 39 (76.47\%), and 38 (74.51\%) cases, respectively. The differentiation rates of the $40 \mathrm{keV}$ monoenergetic, iodine density, and fusion images were significantly higher than those of conventional images $\left(\chi^{2}=-0.35,-0.43\right.$, and -0.41 , respectively, all $\left.\mathrm{P}<0.001\right)$. There were no significant differences in the differentiation rates among the $40 \mathrm{keV}$ monoenergetic, iodine density, and fusion images $\left(\chi^{2}=-0.06,-0.08\right.$, 0.02 , respectively, all $\mathrm{P}=1.00)$. The maximal tumor diameters in the four images did not significantly differ $\left(\chi^{2}=3.61, \mathrm{P}=0.31\right)$. Conventional and spectral images of unenhanced and arterial phases could not/barely identify the tumor borderlines.

Conclusions: Venous-phase spectral images of double-layer spectral detector CT can differentiate most central lung cancers from atelectasis, and the maximal diameter measurement of the tumor is reliable. Double-layer spectral detector CT can accurately identify the borderlines of most central lung cancers through spectral images during routine CT examinations without requiring other imaging modalities. Therefore, this method has considerable clinical value for applications in tumor staging, efficacy evaluation, and radiotherapy.

Keywords: Central lung cancer (CLC); atelectasis; double-layer spectral detector computed tomography (CT) 
Submitted Sep 28, 2021. Accepted for publication Nov 25, 2021.

doi: 10.21037/apm-21-3083

View this article at: https://dx.doi.org/10.21037/apm-21-3083

\section{Introduction}

Central lung cancer (CLC) with obstructive atelectasis is very common in clinical practice. Determination of the tumor borderline is important for tumor staging, resectability assessment, efficacy evaluation, and radiotherapy target delineation $(1,2)$. computed tomography (CT), positron emission tomography/computed tomography (PET-CT), and Magnetic Resonance Imaging (MRI) are currently the most common imaging modalities for tumor diagnoses. However, due to the low soft-tissue resolution, conventional CT alone may not be sufficiently accurate to distinguish CLC from obstructive atelectasis (3). MRI and PET-CT are more commonly used for this type of tumor $(4,5)$. Nevertheless, both of them have some limitations, such as vulnerability to movement, more contraindications for MRI, and high costs and low popularity for PET-CT. With the development of technology, dual-energy spectral computed tomography (DESCT) has been increasingly applied in clinical practice. Compared with MRI and PETCT, DESCT has the advantages of convenience, cheapness and fewer contraindications. DESCT can provide multiple postprocessing spectral images, including virtual monoenergetic images (MonoE), iodine density (ID) maps, effective atomic number maps $\left(Z_{\text {eff }}\right)$, and fusion images, which greatly improve soft-tissue resolution (6). Unlike other dual-energy techniques, double-layer spectral detector computed tomography (DLSDCT) allows the simultaneous measurement of low- and high-energy photons at the exact same spatial and angular location, facilitating dual-energy postprocessing in the projection domain. The remarkable reduction in the noise of spectral images and radiation dose is very convenient for clinical use (7).

In this study, we examined the feasibility of using DLSDCT in differentiating CLC from atelectasis and analyzed the best imaging in various postprocessing spectral functions. To our knowledge, this kind of topic had not been reported. We present the following article in accordance with the MDAR checklist (available at https://apm. amegroups.com/article/view/10.21037/apm-21-3083/rc).

\section{Methods}

\section{Patients}

This study was conducted in accordance with the Declaration of Helsinki (as revised in 2013), and was approved by the institutional ethics committee of Shandong Cancer Hospital and Institute (No. SDTHEC2021001001). Individual consent for this retrospective analysis was waived. The DLSDCT images of patients with pathologically confirmed CLC were retrospectively analyzed. The inclusion criteria were pathologically confirmed CLC with obvious atelectasis and no previous tumor treatment. The exclusion criteria were incomplete DLSDCT images or obvious artifacts.

\section{DLSDCT examinations}

All scans were performed on a clinically available DLSDCT scanner (IQon, Philips healthcare, Best, The Netherlands). The scan protocol included unenhanced chest scans, arterial phase scans, and venous-phase-enhanced scans. The scanning range was from the thoracic entrance to the diaphragm level, including the whole lung field. The contrast agent, iodiazol $(350 \mathrm{mg} / \mathrm{mL}$, Beilu Pharmaceutical Co. Ltd., Beijing, China), was injected with a highpressure bolus injector, with an injection flow velocity of $2.5 \mathrm{~mL} / \mathrm{s}$ and a dose of $80 \mathrm{~mL}$. Arterial and venous scans were performed $30 \mathrm{~s}$ and $60 \mathrm{~s}$ post-injection, respectively. The parameters used for CT scanning were as follows: collimation $0.625 \mathrm{~mm} \times 64$; pitch $1.015 ; 120$ kilovolt peak $(\mathrm{kVp})$; automatic milli-Ampere Times Second (mAs) technology; tube speed $0.5 \mathrm{~s} /$ cycle; reconstruction layer thickness and spacing, $1 \mathrm{~mm}$; and image matrix, $512 \times 512$. The lung (window width/level 1,600/-600 HU) and mediastinal (window width/level 400/40 HU) images were routinely reconstructed.

\section{Imaging analysis}

The three-phase mediastinal images of the unenhanced 
scan, arterial phase, and venous phase were uploaded to the Philips Spectral Diagnostic Suite 9.0 (Philips healthcare, Best, The Netherlands) and postprocessed. Two senior physicians observed the conventional CT and three types of spectral images (40 keV MonoE, ID, and MonoE-ID fusion images) of each phase to determine the differentiation of tumors from atelectasis. Differentiable tumor was defined as a clear tumor boundary that could be clearly delineated with a mouse. If the density difference between the tumor and atelectasis area was mild or there was a fuzzy transition area between them, the tumor with a boundary that could not be clearly delineated was defined as undifferentiable. Disagreements were resolved by consultation. The axial maximal diameter of the tumor was measured for averaging in cases where the tumor borderline was differentiable in all conventional CT and spectral images in one phase.

\section{Statistical analysis}

SPSS 20.0 (SPSS Inc., Chicago, IL, USA) was used for statistical analysis. The maximal tumor diameter was expressed as the mean $\pm \mathrm{SD}$. The rates of differentiating tumors from atelectasis in each image were compared using Cochran's Q test. Dunn's test (Bonferroni correction) was then used for pairwise comparisons. The maximal diameters of the tumors were compared with the Friedman test. A P value $<0.05$ was considered significantly different.

\section{Results}

\section{General clinical information}

A total of 51 patients with pathologically confirmed CLC between Jan. 2020 and Dec. 2020 were enrolled in this study. There were 37 males and 14 females (aged $42-83$ years, with a median age of 63 years), all of whom were complicated with obstructive atelectasis. Twentyseven cases were confirmed by bronchoscopy, 11 cases by operation, nine cases by percutaneous biopsy, and the remaining cases by sputum or pleural effusion cytology. The pathological types were as follows: squamous cell carcinoma in 21 cases, adenocarcinoma in 22 cases, small cell carcinoma in six cases, and other types of malignant tumors in two cases.

\section{Differentiation of tumors from atelectasis in three phases of each image}

Conventional CT, $40 \mathrm{keV}$ MonoE, ID, and MonoE-ID fusion images of unenhanced scans could not effectively identify the tumors and atelectasis. The tumor and atelectasis areas were identified by conventional CT, $40 \mathrm{keV}$ MonoE, ID, and MonoE-ID fusion images in the arterial phase in $4(7.84 \%), 5(9.80 \%), 5(9.80 \%)$, and $6(11.76 \%)$ cases, respectively. Overall, $6(11.76 \%)$ cases were differentiable in the arterial phase. In the conventional arterial phase CT images, all cases showed high enhancement, with adenocarcinoma occurring in five cases and typical carcinoid tumors occurring in one case.

The tumor and atelectasis areas were identified by conventional CT images and $40 \mathrm{keV}$ MonoE, ID, and MonoE-ID fusion images in the venous phase in 17 (33.33\%), 35 (68.63\%), 39 (76.47\%), and $38(74.51 \%)$ cases, respectively (Figure 1). The Cochran's Q results showed that the differentiation rates of tumors from atelectasis areas were significantly different among the four images $\left(\chi^{2}=52.40\right.$, $\mathrm{P}<0.001)$. Pairwise comparison with Dunn's test (Bonferroni correction) indicated that the differentiation rates of $40 \mathrm{keV}$ MonoE, ID, and MonoE-ID fusion images were significantly higher than those of conventional CT images $\left(\chi^{2}=-0.35,-0.43,-0.41\right.$, respectively, all $\mathrm{P}<0.001$ after correction). There were no significant differences in the differentiation rates of $40 \mathrm{keV}$ MonoE, ID, and MonoEID fusion images $\left(\chi^{2}=-0.06,-0.08,0.02\right.$, respectively, all $\mathrm{P}=1.00)$ (Table 1).

\section{Comparison of the maximal tumor diameters}

There were 17 cases that were differentiable among all of the conventional CT, $40 \mathrm{keV}$ MonoE, ID, and MonoE-ID fusion images in the venous phase. The maximal diameters of the tumors in the four images were $50.00 \pm 22.66$, $49.52 \pm 23.10,49.63 \pm 22.97$, and $49.60 \pm 23.07 \mathrm{~mm}$, respectively. The Friedman test showed no significant difference in the maximal diameters of the tumors in the four images $\left(\chi^{2}=3.61\right.$, $\mathrm{P}=0.31$ ).

\section{Discussion}

In this study, we analyzed the application value of DLSDCT in differentiating CLC from atelectasis. The differentiation rates of tumors from atelectasis were retrospectively analyzed using conventional CT and three types of spectral images of unenhanced scans as well as arterial and venous phases. We found that none of these unenhanced scan images could effectively distinguish the tumor and atelectasis areas; only $11.76 \%$ of tumors could be distinguished in 

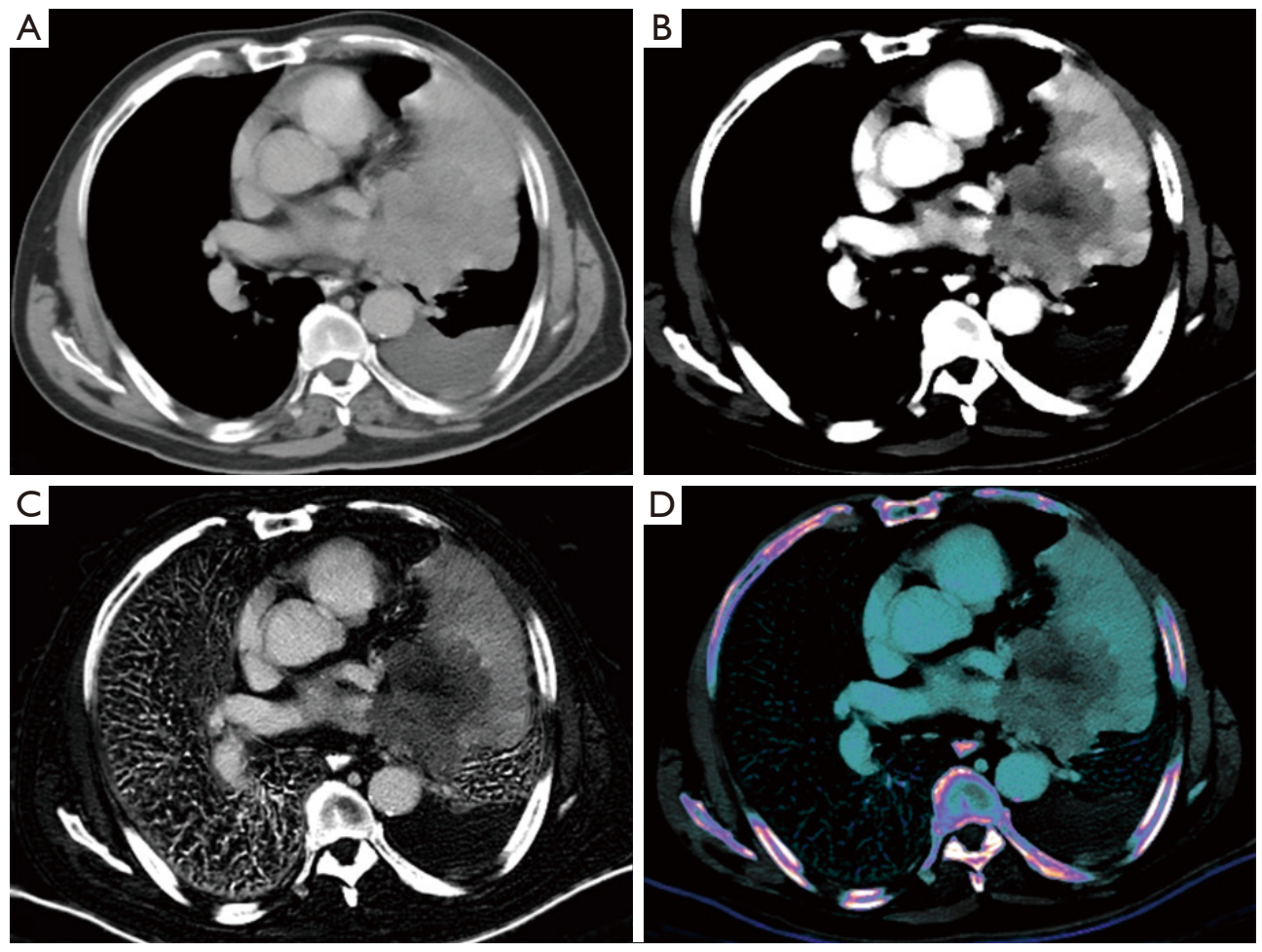

Figure 1 Differentiation of tumors from atelectasis in four types of images in the venous phase. A 69-year-old male with poorly differentiated squamous cell carcinoma and atelectasis. In the venous phase, conventional CT (A) failed to identify the tumor boundary and atelectasis. However, this was possible when using $40 \mathrm{keV}$ virtual monoenergetic (MonoE) images (B), ID maps (C), and MonoE-ID fusion images (D). ID, iodine density.

Table 1 Pairwise comparison of the differentiation rates of tumors from atelectasis in four types of images in the venous phase

\begin{tabular}{lcc}
\hline Pairwise comparison & $\chi^{2}$ & $P$ \\
\hline CT vs. MonoE & -0.35 & 0.00 \\
CT vs. ID & -0.43 & 0.00 \\
CT vs. MonoE-ID & -0.41 & 0.00 \\
MonoE vs. ID & -0.08 & 1.00 \\
MonoE vs. MonoE-ID & -0.06 & 1.00 \\
ID vs. MonoE-ID & 0.02 & 1.00 \\
\hline
\end{tabular}

$\mathrm{CT}$, conventional CT images in the venous phase; MonoE, $40 \mathrm{keV}$ virtual monoenergetic image; ID, iodine density map; MonoE-ID, fusion image of $40 \mathrm{keV}$ MonoE and ID.

the arterial phase. Therefore, except for a few tumors enhanced obviously in the arterial phase, conventional $\mathrm{CT}$ and the three types of spectral images of unenhanced scan and arterial phase were hardly useful in identifying tumor boundaries. Among 51 cases, conventional CT,
$40 \mathrm{keV}$ MonoE, ID, and MonoE-ID fusion images of the venous phase were successful in differentiating tumors from atelectasis in 17 (33.33\%), 35 (68.63\%), 39 (76.47\%), and $38(74.51 \%)$ cases, respectively. The results showed that the venous phase was more efficient in identifying tumor boundaries, which was consistent with the findings of Gao et al. (8). It was speculated that the contrast agent had fully penetrated into the tumor in the venous phase. As the iodine content difference between the tissues increased, the tumor boundary became clearer. However, our study showed that the differentiation rates of tumors from atelectasis were lower than those in other similar studies (8). A possible reason is that our definition of differentiable tumors was stricter. For example, if there is a density difference between a tumor and an atelectasis area but there is a fuzzy transition area between them, the tumor that cannot be clearly delineated is defined as undifferentiable.

DLSDCT can provide $161 \mathrm{MonoE}$ images (from 40 to $200 \mathrm{keV}$ ). Studies have shown that MonoE with low $\mathrm{keV}$ increases the detectability of inconspicuous hilar lymph 
nodes and osteoblastic metastases $(9,10)$ because MonoE with low $\mathrm{keV}$ provides an increased contrast-to-noise ratio (11) and contrast enhancement of vessels, even if the scan is not performed during the early enhancement phase (12). However, in other DESCTs, MonoE with too low keVs exhibits a high noise level and, consequently, an impaired image quality (13). In contrast, DLSDCT facilitates the simultaneous measurement of spatially and temporally perfectly aligned high- and low-energy projection datasets, and thus, can utilize the noise anti-correlation between the detector layers for noise suppression. A previous study indicated that the image noise remains relatively low over the whole energy spectrum from 40 to $200 \mathrm{keV}$ (14). Therefore, the $40 \mathrm{keV} \mathrm{MonoE}$ image recommended by the expert consensus (7) was used for tumor observation in this study. In addition, ID could reflect microvessel density and blood supply, and could serve as a biomarker of tumor vascularity and help to correctly measure the degree of pulmonary nodule enhancement $(15,16)$. Therefore, ID may be sensitive to the enhancement difference between CLC and atelectasis. Also, some researchers found that the default window width/level of the DLSDCT MonoE image was not suitable, affecting observation (17). In the practical process, we also found that the anatomical structures of $40 \mathrm{keV}$ MonoE, ID, and MonoE-ID fusion images were difficult to identify when using the system default width/level, especially the ID image, which often requires manual adjustment but does not have an impact on the tumor measurement. Therefore, we suggest that the DLSDCT system can be properly optimized in the default window width/level settings.

Currently, the application of MRI and PET-CT in differentiating tumors from atelectasis has become a research hotspot $(4,5)$. Studies have reported that the differentiation rate of tumors and atelectasis by MRI T2 weighted image (T2WI) and diffusion weighted image (DWI) is higher than $80 \%$ (18). PET-CT can easily distinguish tumor and atelectasis areas (19), and the delineation of tumor volume by MRI and PET$\mathrm{CT}$ is more accurate than that by conventional dynamic enhanced CT (20). However, MRI and PET-CT still have some limitations, among which the low spatial resolution is difficult to overcome. For example, due to the partial volume effect, there is a deviation of 7-9 $\mathrm{mm}$ between the PET and the real boundary of the tumor (21), inevitably reducing the accurate definition of the PET-CT fusion image's tumor delineation. Moreover, vulnerability to movement, contraindications of MRI, as well as high costs and low popularity of PET-CT restrict their clinical usage. In this study, we found that the differentiation rate of the three types of spectral images in the venous phase, especially ID and MonoE-ID fusion images, was as high as approximately $75 \%$, and the measurement of the maximal tumor diameter was reliable. In addition, the excellent spatial resolution of CT images suggested that spectral images in the venous phase were sensitive and accurate in identifying tumors and atelectasis, thus indicating great clinical application value.

This study has a few limitations that should be noted. Firstly, we only observed the differentiation rate of CLC from atelectasis by DLSDCT in this study, so the strengths and weaknesses of DLSDCT compared with other imaging modalities, such as MRI or PET-CT, remain unclear. Secondly, in addition to the spectral images we observed in this study, DLSDCT can also provide another pseudocolor spectral image, the $Z_{\text {eff }}$ image. In the practical process, we found that $Z_{\text {eff }}$ images were more sensitive to the difference between tissues, and that inhomogeneity or necrosis within the tumor could be marked with different colors. However, $Z_{\text {eff }}$ images are greatly affected by the window width/level settings, so it is difficult to determine the tumor boundary with $Z_{\text {eff }}$ images alone. Therefore, this study did not involve $Z_{\text {eff }}$-related content, which will be investigated in the future. Thirdly, similar to other studies, the reliability of the tumor boundary delineated by DLSDCT remains unclear because the true boundary is difficult to confirm. Finally, this study was retrospective and the sample size was small. So multicenter clinical trials with larger sample will be performed later.

\section{Conclusions}

In this study, we confirmed that the spectral images in the venous phase of DLSDCT could distinguish most CLCs from atelectasis, with highly consistent tumor measurements. DLSDCT could accurately identify the boundaries of most CLCs with spectral postprocessing images in routine CT examinations without the need for other imaging modalities; thus, it has great clinical value for applications in tumor staging, efficacy evaluation, and radiotherapy.

\section{Acknowledgments}

Funding: This work was supported by the Shandong Medical and Health Science and Technology Development 
Project (No. 2019WS200 to YGQ, QZ, and MXF).

\section{Footnote}

Reporting Checklist: The authors have completed the MDAR checklist. Available at https://apm.amegroups. com/article/view/10.21037/apm-21-3083/rc

Data Sharing Statement: Available at https://apm. amegroups.com/article/view/10.21037/apm-21-3083/dss

Conflicts of Interest: All authors have completed the ICMJE uniform disclosure form (available at https://apm. amegroups.com/article/view/10.21037/apm-21-3083/coif). All authors report the language of this work was edited by AJE. This work was supported by the Shandong Medical and Health Science and Technology Development Project (No. 2019WS200 to YGQ, QZ, and MXF). The authors have no other conflicts of interest to declare.

Ethical Statement: The authors are accountable for all aspects of the work in ensuring that questions related to the accuracy or integrity of any part of the work are appropriately investigated and resolved. This study was conducted in accordance with the Declaration of Helsinki (as revised in 2013), and was approved by the institutional ethics committee of Shandong Cancer Hospital and Institute (No. SDTHEC2021001001). Individual consent for this retrospective analysis was waived.

Open Access Statement: This is an Open Access article distributed in accordance with the Creative Commons Attribution-NonCommercial-NoDerivs 4.0 International License (CC BY-NC-ND 4.0), which permits the noncommercial replication and distribution of the article with the strict proviso that no changes or edits are made and the original work is properly cited (including links to both the formal publication through the relevant DOI and the license). See: https://creativecommons.org/licenses/by-nc-nd/4.0/.

\section{References}

1. Akhurst T. Staging of Non-Small-Cell Lung Cancer. PET Clin 2018;13:1-10.

2. Eradat J, Abtin F, Gutierrez A, et al. Evaluation of treatment response after nonoperative therapy for early-stage non-small cell lung carcinoma. Cancer J 2011;17:38-48.
3. Tobler J, Levitt RG, Glazer HS, et al. Differentiation of proximal bronchogenic carcinoma from postobstructive lobar collapse by magnetic resonance imaging. Comparison with computed tomography. Invest Radiol 1987;22:538-43.

4. Flechsig P, Rastgoo R, Kratochwil C, et al. Impact of Computer-Aided CT and PET Analysis on Non-invasive T Staging in Patients with Lung Cancer and Atelectasis. Mol Imaging Biol 2018;20:1044-52.

5. Yang RM, Li L, Wei XH, et al. Differentiation of central lung cancer from atelectasis: comparison of diffusionweighted MRI with PET/CT. PLoS One 2013;8:e60279.

6. Rassouli N, Etesami M, Dhanantwari A, et al. Detectorbased spectral CT with a novel dual-layer technology: principles and applications. Insights Imaging 2017;8:589-98.

7. Chinese Journal of Radiology Cooperative Group of Clinical Application of Dual-layer Spectral Detector CT. China expert consensus on clinical application of duallayer spectral detector CT. Chinese Journal of Radiology 2020;54:635-43.

8. Gao SY, Chen Y, Li XT, et al. single source dual energy CT differentiation of central lung cancer and pulmonary atelectasis. Chinese Journal of Medical Imaging Technology 2016;32:258-61.

9. Sekiguchi T, Ozawa Y, Hara M, et al. Visibility of the hilar lymph nodes using advanced virtual monoenergetic low$\mathrm{keV}$ images for preoperative evaluation of lung cancer. $\mathrm{Br} \mathrm{J}$ Radiol 2019;92:20180734.

10. Yue D, Ru Xin W, Jing C, et al. Virtual monochromatic spectral imaging for the evaluation of vertebral inconspicuous osteoblastic metastases from lung. Acta Radiol 2017;58:1485-92.

11. Albrecht MH, Scholtz JE, Hüsers K, et al. Advanced image-based virtual monoenergetic dual-energy CT angiography of the abdomen: optimization of kiloelectron volt settings to improve image contrast. Eur Radiol 2016;26:1863-70.

12. Thaiss WM, Sauter AW, Bongers M, et al. Clinical applications for dual energy CT versus dynamic contrast enhanced CT in oncology. Eur J Radiol 2015;84:2368-79.

13. Albrecht MH, Trommer J, Wichmann JL, et al. Comprehensive Comparison of Virtual Monoenergetic and Linearly Blended Reconstruction Techniques in Third-Generation Dual-Source Dual-Energy Computed Tomography Angiography of the Thorax and Abdomen. Invest Radiol 2016;51:582-90.

14. Doerner J, Wybranski C, Byrtus J, et al. Intra-individual comparison between abdominal virtual mono-energetic 
spectral and conventional images using a novel spectral detector CT. PLoS One 2017;12:e0183759.

15. Chae EJ, Song JW, Seo JB, et al. Clinical utility of dualenergy CT in the evaluation of solitary pulmonary nodules: initial experience. Radiology 2008;249:671-81.

16. Jia Y, Xiao X, Sun Q, et al. CT spectral parameters and serum tumour markers to differentiate histological types of cancer histology. Clin Radiol 2018;73:1033-40.

17. Doerner J, Luetkens JA, Iuga AI, et al. Poly-energetic and virtual mono-energetic images from a novel dual-layer spectral detector CT: optimization of window settings is crucial to improve subjective image quality in abdominal CT angiographies. Abdom Radiol (NY) 2018;43:742-50.

18. Qi LP, Zhang XP, Tang L, et al. Using diffusion-weighted MR imaging for tumor detection in the collapsed lung: a

Cite this article as: Wen LJ, Zhao QY, Yin YH, Wang B, Fan MX, Qi YG, Zhang Q. Application value of double-layer spectral detector CT in differentiating central lung cancer from atelectasis. Ann Palliat Med 2022;11(6):1990-1996. doi: 10.21037/ apm-21-3083 preliminary study. Eur Radiol 2009;19:333-41.

19. Ling CC, Humm J, Larson S, et al. Towards multidimensional radiotherapy (MD-CRT): biological imaging and biological conformality. Int J Radiat Oncol Biol Phys 2000;47:551-60.

20. Zhang X, Fu Z, Gong G, et al. Implementation of diffusion-weighted magnetic resonance imaging in target delineation of central lung cancer accompanied with atelectasis in precision radiotherapy. Oncol Lett 2017;14:2677-82.

21. Ford EC, Herman J, Yorke E, et al. 18F-FDG PET/CT for image-guided and intensity-modulated radiotherapy. J Nucl Med 2009;50:1655-65.

(English Language Editor: A. Kassem) 